Ann. Sci. forest., 1980, 37 (3), 239-248.

\title{
Facteurs limitants de la croissance initiale d'une plantation de Merisier (Prunus avium L.) sur rendzine brunifiée
}

\author{
H. FROCHOT * et G. LEVY** \\ avec la collaboration technique de Y. LEFEVRE** ef L. WEHRLEN* \\ * Station de Sylviculfure et de Production \\ ** Station de Recherches sur les Sols Forestiers ef la Fertilisation \\ Centre national de Recherches forestières, I.N.R.A., Champenoux, 54280 Seichamps
}

\begin{abstract}
Résumé
Un essai est réalisé dans une plantation de l'année de Merisier (Prunus avium L.), sur rendzine brunifiée. Différents traitements sont effectués, seuls ou combinés : herbicides, engrais et films plastiques (transparent, noir, opaque thermique).

Au bout de 2 ans, herbicide (métobromuron $2000 \mathrm{~g} /$ ha, puis glyphosate localisé $2160 \mathrm{~g} / \mathrm{ha}$ ) et films plastiques améliorent très nettement la croissance. La fertilisation a un effel favorable seulement lorsqu'elle est combinée au plastique noir (interaction hautement significative). Dans ce cas, l'accroissement en hauteur est multiplié par 2,2 et l'accroissement en section par 6,8 .

On suppose que l'alimentation en eau constitue le facteur limitant primaire de la croissance, la nutrition minérale le facteur limitant secondaire.
\end{abstract}

\section{Introduction}

L'échec d'un certain nombre d'essais de fertilisation de plantations forestières ne signifie pas forcément que les conditions de nutrition minérale sont à leur optimum. II n'esł en effet pas impossible qu'une (ou plusieurs) autre variable constitue le facteur limitant primaire de la croissance dans ces stations. On peut penser notamment à l'alimentation en eau des plants, à la température du sol, à la concurrence herbacée (facteur complexe, mais très important, notamment au démarrage des plants).

Aussi est-il intéressant de déterminer sur divers types de station les facteurs limitants de la croissance des plants ef l'ordre dans lequel ils interviennent. C'est dans cette optique que nous avons entrepris l'essai présenté dans cet article.

Parmi les techniques expérimentales susceptibles de modifier les facteurs de croissance, certaines entraînent des coniraintes matérielles inconciliables avec des essais in situ, éloignés des zones d'activité humaine. Aussi, faut-il utiliser des techniques moins contraignantes, même si leurs effets sont plus complexes à interpréter. Pour ce premier essai, nous nous aidons d'herbicides, d'engrais ef de films plastiques. 
Les films plastiques, qui modifient plusieurs variables du sol, sont d'ailleurs utilisés depuis plusieurs années en cultures maraîchères, en viticulture, en arboriculture fruitière, ainsi que pour la culture du Maïs. Ils sont très favorables dans ce dernier cas, non seulement sur des sols peu colorés comme les rendzines de Champagne (Ballif et Dutil, 1974 et 1975), mais également sur des limons de plateaux (Simon-Sylvestre et Chabannes, 1975). Des essais ont permis de montrer également l'inférêt de cette technique pour des plantations d'arbres d'alignement (I.D.F., 1977), des peupliers et même des essences forestières, dont des résineux (C.E.R.A.F.E.R., 1972 ; A.F.O.C.E.L., 1978).

Notre but essentiel, qui est de comprendre le rôle de plusieurs facteurs du milieu ef leur interaction sur la croissance, ne nous fait pas négliger les intérêts pratiques des améliorations apportées par les techniques mises en œuvre. C'est pourquoi nous avons choisi d'opérer en priorité sur des espèces pour lesquelles l'investissement initial supplémentaire a le plus de chances d'être rentable :

- soit qu'll permette d'éviter ou de réduire un cerlain nombre d'opérations coûteuses après plantation (regarnis, entretiens, dégagements) ;

- soit qu'il entraîne une amélioration suffisante de la croissance pour compenser les dépenses supplémentaires.

Les feuillus à croissance rapide, notamment les feuillus précieux, sont particulièrement concernés, d'autant plus que les plantations de ces essences vont probablement s'intensifier dans le cadre de la mise en valeur des taillis et taillis-sous-futaie de la partie Nord de la France.

Le Merisier (Prunus avium L.) a été choisi pour ce premier essai. Il nous a semblé opportun de présenter les résultats acquis après 2 années seulement de plantation, car les différences de croissances obtenues sont telles qu'elles ne peuvent pas être remises en cause par l'évolution ultérieure de l'essai.

\section{1. - Méthodologie}

\section{1. - Implantation}

L'expérience est située en Lorraine, à proximité de Foug (Meurthe-et-Moselle), dans une forêt particulière, à la place d'un ancien taillis-sous-futaie pauvre en bordure de zone de culture. La parcelle a été dessouchée et parfaitement mise à nu par une lame Rome au cours de l'hiver précédent la plantation. L'endroit est exposé au vent.

On se trouve sur plateau calcaire, de pente pratiquement nulle, sur argovien; le sol peu épais va de la rendzine brunifiée (surtout) au sol brun calcaire ; les cailloux calcaires, déjà présents en surface, sont très abondants à partir de $30 \mathrm{~cm}$. L'humus est un mull calcique à texture argileuse et de $\mathrm{pH} \mathrm{7,6;} \mathrm{il} \mathrm{contient} 0,10$ p. 1000 de phosphore assimilable (méthode Duchaufour) et, en milliéquivalents pour $100 \mathrm{~g}, 50,4$ de calcium, 0,7 de potassium et 1,4 de magnésium.

\section{2. - Matériel végétal}

L'essai a été installé dans une plantation de l'année de merisiers $(1+0)$ issus de semis élevés en pépinière d'environ $25 \mathrm{~cm}$ de hauteur moyenne ; l'écartement des plants est de $2 \times 2,75 \mathrm{~m}$; la plantation a été réalisée du 25 au 30 mars 1977 . 


\section{3. - Traitements simples}

Les traitements suivants ont été appliqués, seuls ou combinés :

Fertilisation.

Fumure complète le 28 avril 1977 par épandage suivi d'un enfouissement superficiel par griffage sur un rayon de $25 \mathrm{~cm}$ autour de chaque plant.

Dose individuelle : $60 \mathrm{~g}$ de superphosphate triple $(11,7 \mathrm{~g}$ de $\mathrm{P}), 50 \mathrm{~g}$ de patentkali (sulfate double de potassium et de magnésium ; $11,7 \mathrm{~g}$ de $\mathrm{K}$ et $2,4 \mathrm{~g}$ de $\mathrm{Mg}$ ) et $10 \mathrm{~g}$ d'ammonitrate (3 $\mathrm{g}$ de $\mathrm{N})$.

Herbicide.

En prévision de la combinaison des modalités herbicides et films plastiques, on a besoin d'un herbicide persistant de façon à pouvoir l'appliquer avant l'installation du film. Peu de données existent sur Prunus avium ; on a choisi un herbicide sélectif vis-àvis de Prunus mahaleb, le métobromuron (Carré et Barralis, 1973).

Dans les modalités « herbicide non combiné au plastique », par contre, le métobromuron risquait d'être insuffisamment efficace sur les végétaux pérennes qui peuvent se réinstaller. L'application d'un autre herbicide, le glyphosate, a donc été prévue en complément du mélobromuron pour les maîtriser.

Métobromuron, herbicide du groupe des urées substituées ; utilisé à $2000 \mathrm{~g} / \mathrm{ha}$ de matière active, en bouillie aqueuse à $1000 \mathrm{l} / \mathrm{ha}$ :

- absorbé par les plantules, il inhibe la photosynthèse d'un grand nombre de mono- et dicotylédones herbacées ;

- pulvérisation généralisée sur une surface égale à celle des films plastiques (environ $2 \mathrm{~m}^{2}$ ) le 29 avril 1977.

Glyphosate ou acide (phosphonométhylamino)-2-acétique ; utilisé à $2160 \mathrm{~g} / \mathrm{ha}$ de matière active, en bouillie aqueuse à $1500 \mathrm{l} / \mathrm{ha}$;

- systémique absorbé par les parties aériennes chlorophylliennes surtout, il est efficace sur les espèces pérennes herbacées bien développées, et maîtrise un certain nombre d'espèces ligneuses ;

- pulvérisation dirigée autour des plants sur une surface égale à celle des films plastiques, le 6 juillet 1977.

Paillage plastique.

On utilise des découpes de plastique de $1,80 \times 1,10 \mathrm{~m}$, perforées au centre d'un trou de $4 \mathrm{~cm}$ de diamètre pour le passage du plant. Les bords sont recouverts de terre pour maintenir le film en place.

Trois types différents de plastique (polyéthylène basse densité) ont été utilisés ; leurs caractéristiques et effets principaux sont les suivants (Buclon, 1971) :

Plastique transparent.

Epaisseur $80 \mu$.

- économie de l'eau*,

* Sous les films, le sol est presque toujours plus humide qu'en sol nu (suppression de l'évaporation), sauf pendant un temps limité après certaines pluies. 
- augmentation de la température de surface du sol,

- favorise les mauvaises herbes tant que la température est peu élevée, puis provoque leur dessèchement lorsqu'elle s'élève.

Plastique noir.

Epaisseur $100 \mu$.

- économie de l'eau,

- effet herbicide par suppression totale de la lumière,

- une certaine augmentation de la température du sol, surtout de nuit, mais inférieure à celle du plastique transparent.

Opaque thermique.

Epaisseur $50 \mu$. visibles,

- théoriquement transparent aux infra-rouges et opaque aux longueurs d'onde

- effet intermédiaire entre les deux types précédents.

Les films plastiques ont d'autres effets, généralement bénéfiques, sur la structure du sol, sa teneur en sels minéraux, la rétention du $\mathrm{CO}_{2}$, l'enracinement.

1. - Témoin (T).

\section{4. - Modalités de l'essai}

2. - Herbicide $(H)$ : métobromuron, puis glyphosate.

3. - Fertilisation (E).

4. - Herbicide - fertilisation (HE); mêmes traitements herbicides que $(H)$.

5. - Film plastique transparent $(P)$.

6. - Film plastique noir (N).

7. - Film plastique opaque thermique (O).

8. - Film plastique transparent + herbicide (PH), métobromuron uniquement.

9. - Film plastique transparent + fertilisation (PE).

10. - Film plastique noir + fertilisation (NE).

11. - Film plastique opaque thermique + fertilisation (OE).

12. - Film plastique transparent + fertilisation + herbicide (PHE) ; métobromuron uniquement.

Nous avons jugé inutile d'introduire d'autres modalités, pour ne pas alourdir encore l'essai, et pour des raisons plus spécifiques :

- métobromuron seul : les insuffisances d'efficacité de cet herbicide sur les végétaux pérennes en place ne sont plus à démontrer;

- glyphosate sous plastique : l'époque de traitement, située bien après la mise en place des plastiques dans les conditions de l'essai, rendait la pratique de l'opération peu réaliste ;

- arrosage, sarclage : ces modalités sont à réserver pour des essais plus accessibles ef plus facilement contrôlables.

\section{5. - Dispositif expérimental}

Essai en blocs à dix répétitions monoarbres, sur des sujets sains choisis dans une tranche de hauteur initiale de 20 à $30 \mathrm{~cm}$. 


\section{2. - Résultats}

\section{1. - Effets des traitements sur la végétation indésirable}

Dans les témoins, suite à la préparation du sol, de rares rejets de feuillus (Cornus mas surtout) et une végétation herbacée assez peu variée recouvrent 60 à 70 p. 100 du sol au mois de juillet 1977. Ce n'est qu'au printemps suivant que le sol est totalement recouvert. Les espèces principales sont soit un tapis de Brachypodium sylvaticum, soit un ensemble de dicotylédones où dominent Sonchus asper surtout, mais aussi Coronilla varia, Epilobium angustifolium, Stachys sylvatica, Viola tricholor.

Dans les modalités herbicides ( $\mathrm{H}$ ef $\mathrm{HE}$ ), le taux de « mauvaises herbes » est assez faible : au printemps 1977, il reste des espèces pérennes pour lesquelles le métobromuron a été sans effet (Brachypodium sylvaticum surtout). Par contre, après le traitement glyphosate, et jusqu'à la fin de l'année 1978, le sol reste propre, avec seulement quelques espèces assez insignifiantes.

Sous plastique noir ( $N$ et $N E$ ), aucune végétation ne s'est développée.

Sous film transparent ( $P$ et $P E)$ par contre, une végétation semblable au témoin s'est développée jusqu'au mois de juin de chacune des deux années ; elle a été ensuite brôlée par le soleil et s'est desséchée (juin-juillet).

Sous opaque thermique ( $O$ et $O E$ ), la végétation s'est développée de façon assez comparable au plastique transparent.

L'herbicide sous paillage transparent ( $\mathrm{PH}$ et $\mathrm{PHE}$ ) a été très efficace, sauf sur pérennes déjà en place. Le désherbage est meilleur qu'en $P$ et $H$ en juin 1977.

L'apport d'engrais, seul ou combiné (avec $\mathrm{H}, \mathrm{O}, \mathrm{P}$ ou $\mathrm{PH}$ ) n'a jamais modifié sensiblement les caractéristiques de la végétation.

\section{2. - Effet sur les merisiers}

La reprise a été très bonne dans tous les traitements. Un quari des plants des traitements sans plastique ont été broutés par des chevreuils, alors que ce n'est le cas d'aucun des plants entourés de plastique. Notons également une appétence particulière possible du chevreuil pour les plants des modalités herbicides ( $H$ et $H E$ ).

Les divers accroissements selon les traitements sont présentés sur la figure 1.

L'étude statistique des interactions a par ailleurs donné les résultats suivants :

- Interactions négatives.

$\mathrm{P} \times \mathrm{H}:-$ Interaction significative sur l'accroissement en hauteur 1977, 78 ef total $(77+78)$.

- Interaction presque significative (entre 5 ef 10 p. 100) sur l'accroissement en diamètre 77 et total.

- Interaction positive.

$\mathrm{N} \times \mathrm{E}:-$ Interaction très hautement significative sur l'accroissement en diamètre 78 , et presque significative (10 p. 100) sur l'accroissement en diamètre total. 

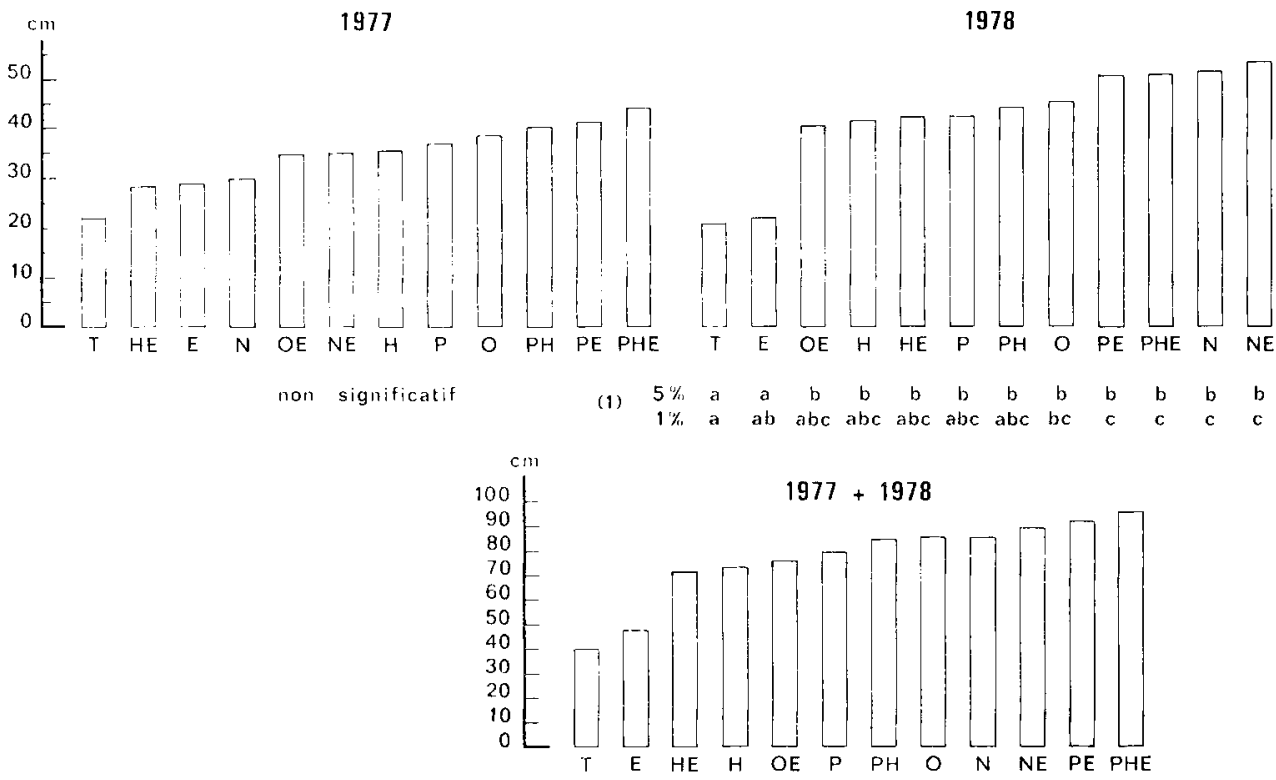

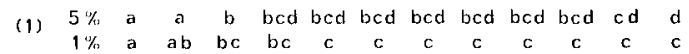

Accroissements en diamètre
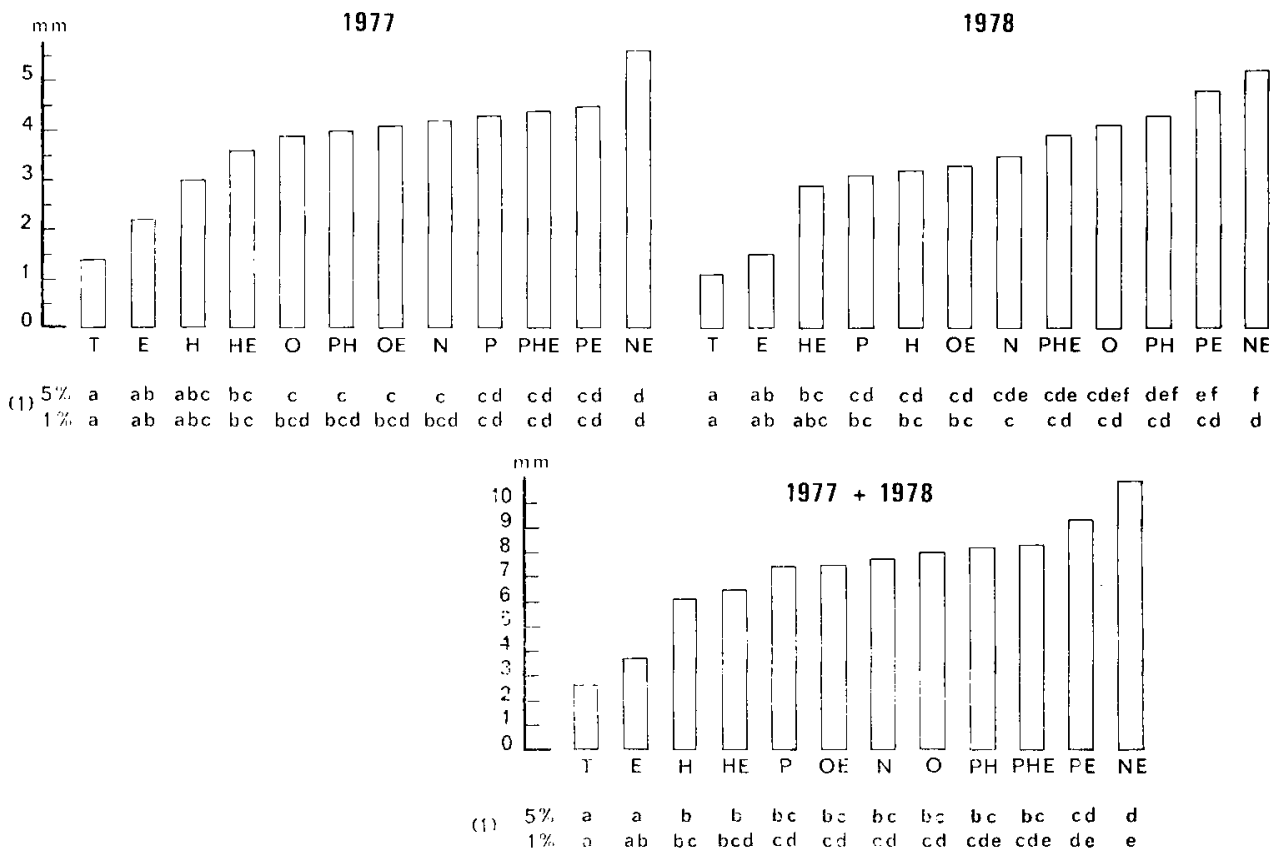

FIG. 1. - Accroissement de jeunes Prunus avium soumis à différents traitements.

Increment of young wild cherry (Prunus avium L.) trees with different treatments.

N.B. (1) Les traitements qui n'ont pas au moins une lettre commune (a, b...)

sont statistiquement différents.

Treatments which do not have at least one letter $(a, b, \ldots)$ in common are statistically different. 


\section{3. - Discussion et conclusions}

\section{Croissance en diamètre}

La modalité «herbicide » et chaque type de film plastique améliorent très nettement la croissance en diamètre, alors que la fertilisation est sans effet. Par contre, lorsque cette dernière est combinée au plastique noir, elle a une action positive significative, principalement en $2^{e}$ année.

La nutrition minérale apparaît donc comme un facteur limitant secondaire de la croissance en diamètre dans cette station ; elle n'a pu être améliorée par l'application d'engrais qu'après suppression du facteur limitant primaire par le film plastique noir. Or ce dernier, outre son effet direct sur la rétention de l'eau, a été la seule modalité à supprimer parfaitement la végétation concurrente, ce qui a permis une disponibilité en eau maximale pour le plant.

L'interaction négative $\mathrm{H} \times \mathrm{P}$ laisse également supposer que l'effet favorable de l'herbicide dans cet essai est dû principalement à l'amélioration de l'alimentation des plants en eau.

II n'existe pas d'interaction positive entre la fertilisation el les autres types de film $(\mathrm{P}$ et $\mathrm{O})$, sans doute en raison de la présence de mauvaises herbes en début de saison de végétation : l'amélioration de l'alimentation en eau obtenue par ces plastiques, comme celle obtenue par l'herbicide non combiné au plastique, serait encore insuffisante pour permettre une action positive de l'engrais.

L'hypothèse la plus vraisemblable est donc la suivante : le facteur limitant essentiel de la croissance en diamètre des merisiers a été l'alimentation en eau ; la nutrition minérale, facteur limiłant secondaire, n'a pu être améliorée que lorsque l'alimentation en eau a été suffisante.

\section{Croissance en hauteur}

Les résultats concernant l'accroissement en diamètre sont plus fiables que ceux des hauteurs, en raison des accidents divers survenus sur les jeunes pousses (gelées fardives, chevreuil). II a même fallu supprimer de l'analyse des plants broutés plusieurs fois de suite pour lesquels la pousse de la première année était à peu près nulle. Les croissances en hauteur 1977 n'offrent ainsi guère d'intérêt (les différences ne sont d'ailleurs pas significatives).

Les résultats de l'accroissement en hauteur 1978 sont assez comparables à ceux du diamètre, en ce sens que tous les traitements, sauf $E$, l'améliorent significativement, ef qu'on retrouve l'interaction négative $\mathrm{P} \times \mathrm{H}$.

Par contre, on ne retrouve plus l'interaction $N \times E$ bien que les trois meilleurs traitements combinent film plastique noir ou transparent avec la fertilisation.

\section{Aspect pratique}

Sur un plan plus pratique, deux traitements peuvent apporter des améliorations particulièrement intéressantes : 
- soit l'utilisation d'herbicide $(\mathrm{H})$ qui a permis de multiplier l'accroissement en hauteur par 1,8 et en section par 3,1 ;

- soit la combinaison plastique noir el fertilisation (NE), qui a permis de multiplier la pousse par 2,2 ef l'accroissement en section par 6,8 .

Le premier, $\mathrm{H}$, a l'avantage d'être applicable dans n'importe quelle condition de station, mais demande une étude plus approfondie du désherbage chimique du merisier. Le second nécessite des conditions bien particulières d'installation : le sol doit être bien préparé, sans trop de souches ni de rejets de feuillus ou de ronces. Les films plastiques occasionnent également une gêne pour le passage des engins mécaniques lors des entretiens entre les lignes; toutefois, ceux-ci sont rendus moins fréquemment nécessaires du fait du gain de croissance. D'autre part, le gros gibier (sanglier surtout) soulève volontiers les films plastiques; sa présence éventuelle doit être prise en considération dans les critères d'utilisation. Notons cependant à ce sujet que l'introduction d'essences naturellement peu fréquentes, ou de techniques particulières, a souvent pour effet de favoriser les visites du gibier et de faciliter ses prédations.

Au niveau du coût, le choix est le suivant : l'herbicide nécessite un investissement minimum, à établir sur plusieurs passages pendant les premières années de la plantation. La combinaison plastique noir + engrais correspond à un investissement initial plus élevé, mais permet d'économiser sur les premiers entretiens ; l'engrais entre pour une faible part dans le coût de cette opération.

Enfin, ces résultats spectaculaires, obtenus pendant les deux premières années de la plantation, ne permettent pas de préjuger de la croissance des plants au cours des prochaines années. Par ailleurs, les enseignements obtenus ne sont applicables qu'à des stations de même type.

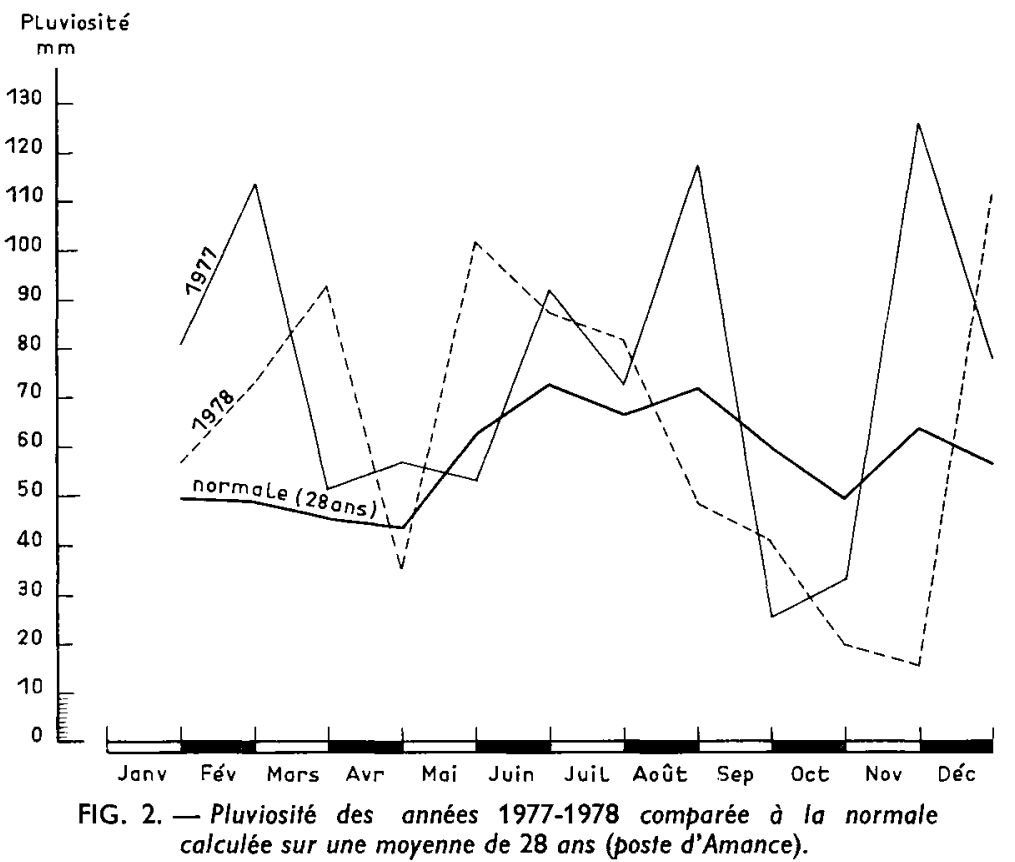

Precipitation for the years 1977-1978 compared with the mean annual precipitation (mean is the average for 28 years) (Amance weather station). 


\section{Conditions climatiques}

On ne connaît pas la part que prennent les conditions climatiques annuelles dans les résultats. Ceux-ci sont en fait une somme de composantes unitaires instantanées qui s'additionnent ef qui peuvent varier selon le climat (eau et température surtout), et selon la région.

La pluviosité des années 77-78 (fig. 2), relevée au poste d'Amance, situé à $40 \mathrm{~km}$ à l'est, a été nettement plus forte que la normale. Elle se caractérise notamment par :

1) la présence d'une certaine réserve en eau dans le sol au début du printemps, à la suite d'un hiver très humide ( 70 p. 100 supérieur à la normale en 77 et 55 p. 100 en 78 pour la période de janvier à mars) (tabl. 1) ;

2) des précipitations supérieures à la normale entre avril et juillet (12 p. 100 de plus en 77 et 25 p. 100 en 78 pour ces 4 mois).

En 1978, les merisiers avaient déjà cessé leur croissance en diamètre et en hauteur le 19 juillet. Les gains d'accroissement ont donc été obtenus dans une période courte ( 3 mois maximum), pendant laquelle la disponibilité en eau a été relativement bonne. Ces résultats laissent supposer que l'influence du paillage plastique aurait été encore plus marquée, par rapport au témoin, une année plus proche de la normale (moins pluvieuse entre avril et juillet).

\section{TABLEAU 1}

Pluviosité des mois de janvier à juillet, en p. 100 por rapport à la normale calculée sur 28 ans Monthly precipitation (January to July) in percent of the mean monthly precipitation (the mean is the overage for 28 years)

\begin{tabular}{|c|c|c|c|c|c|c|c|}
\hline Années & Janvier & Février & Mars & Avril & Mai & Juin & Juillet \\
\hline $\begin{array}{l}1977 \\
1978\end{array}$ & $\begin{array}{r}+63 \text { p. } 100 \\
+15 \text { p. } 100\end{array}$ & $\begin{array}{l}+132 \text { p. } 100 \\
+49 \text { p. } 100\end{array}$ & $\begin{array}{l}14 \text { p. } 100 \\
+\quad 105 \text { p. } 100\end{array}$ & $\begin{array}{r}29 \text { p. } 100 \\
-19 \text { p. } 100\end{array}$ & $\begin{array}{l}-15 \text { p. } 100 \\
+63 \text { p. } 100\end{array}$ & $\begin{array}{l}+27 \text { p. } 100 \\
+21 \text { p. } 100\end{array}$ & $\begin{array}{r}+10 \text { p. } 100 \\
+23 \text { p. } 100\end{array}$ \\
\hline
\end{tabular}

Le fait de laisser en place les films plastiques plusieurs années pose le problème de la reconstitution des réserves en eau du sol au cours de l'hiver. Cet aspect sera étudié par la suite. Néanmoins, nous pouvons déjà faire deux constatations :

1) Le gain de croissance sous les films plastiques a été globalement semblable en 1 re el en $2^{\mathrm{e}}$ année.

2) Des mesures d'humidité réalisées dans un autre de nos essais ont montré que les réserves s'étaient parfaitement reconstituées sous film plastique la $3^{e}$ année après installation.

Les résultats très encourageants de cette première expérience nous incitent à utiliser la même méthode dans d'autres types de stations. A l'avenir, des mesures périodiques concernant les paramètres pris en compte devraient permettre une meilleure compréhension de leur influence réelle sur la croissance des jeunes plants. Nous comptons également déterminer l'effet à plus long terme et la durée utile d'application des différentes modalités (notamment le paillage plastique). 
Enfin, cet essai montre - une fois de plus - l'importance de la concurrence qu'exerce la végéfation herbacée vis-à-vis des jeunes arbres, et la nécessité de poursuivre les études que nous avons déjà engagées dans ce domaine.

Reçu pour publication en octobre 1979.

\section{Remerciements}

Nous tenons à exprimer nos remerciements à M. Hanras, ingénieur aux «Plastiques de Carmaux ", qui nous a fourni gracieusement les films plastiques, à M. Bodin, Technicien au C.R.P.F. Lorraine-Alsace pour l'aide matérielle qu'il nous a apportée lors de l'installation de l'essai, ainsi qu'à M. Hugot, propriétaire de la parcelle.

\section{Summary}

Limiting factors of the juvenile growth of a wild cherry (Prunus avium L.) plantation on a calcareous soil

A trial has been conducted in a wild cherry plantation on a rendzina soil (of the type « rendzine brunifiée »). Different site preparation treatments, simple or combined, are performed : herbicides, fertilizers and plastic film (clear, black, opaque thermic) are used.

After 2 years, herbicides (first metobromuron at $2000 \mathrm{~g} /$ ha and then locally sprayed glyphosate at $2160 \mathrm{~g} / \mathrm{ha}$ ) as well as plastic films improved largely tree growth. Fertilization had a beneficial effect only when combined with black plastic films (the interaction is highly significant) ; in this latter case the height increment of trees is multiplied by 2.2 and the cross section area increment of trees is multiplied by 6.8 .

Water supply is supposed to be the primary limiting factor of tree growth, and mineral nutrition the secondary limiting factor.

\section{Références bibliographiques}

A.F.O.C.E.L.-A.R.M.E.F., 1978. Le paillage plastique des plantations forestières. Informations-Forêt, no 3-1978, 155-163.

BALLIF J. L., DUTIL P., 1974. Utilisation des films plastiques photodégradables pour la culture du maïs en Champagne crayeuse. C. R. Acad. Agric., 60, 389-401.

BALLIF J. L., DUTIL P., 1975. Le réchauffement des sols de craie par films plastiques. Mesures et bilans thermiques. Ann. agron., 26 (2), 159-167.

CARRE R., BARRALIS G., 1973. Contribution à l'éfude du désherbage des pépinières fruitières de porte-greffes. C. R. des journées sur les herbicides, Columa, 13-14 déc. 1973, tome III, 736-743.

BUCLON F., 1971. Bilan de 10 années de recherche et d'application de paillage plastique en France ef dans le monde. Plasticulture, no 10,13-30 et no 11, 10-38.

C.E.R.A.F.E.R.-C.T.F., 1972. Influence du paillage au moyen d'un film de polyéthylène noir sur la survie et la croissance d'une plantation de Pin laricio. Rev. Forest. Franç., no 1, 47-50.

I.D.F., 1977. Guide pratique pour la création, la rénovation, la conduite et l'entretien des haies et brise-vent. Bulletin de vulgarisation forestière 77-8, 3-45.

SIMON-SYLVESTRE G., CHABANNE J., 1975. Incidences de quelques techniques culturales sur certaines propriétés du sol : répercussions sur une culture de Maïs. Ann. agron., 26 (1), 75-99. 